\title{
CHOICE OF CONTINUING EDUCATION EVENT IS DEPENDANT UPON TYPE OF PRACTICE
}

Kim Turner MD, Elizabeth VanDenKerkhof DrPH, Mui Lam PhD, William Mackillop MD Departments of Anesthesiology and Community Health and Epidemiology, Queen's University, Kingston, Ont., Canada

INTRODUCTION: There are a wide variety of Continuing Education events (CEE) and information resources (IR) available to Canadian Anesthesiologists. There may be differences in the selection of CEE and IR by anesthesiologists practicing in institutions with and without anesthesia training programs. The purpose of this study was to examine possible differences in the CEE attended and IR accessed, for a specific topic area, between anesthesiologists in institutions with and without anesthesia training programs.

METHODS: Canadian anesthesiologists were sent a postal questionnaire examining the perioperative care of patients with Obstructive Sleep Apnea (OSA). Respondents were also asked questions regarding the CEE attended in the past two years and information resources accessed for information on OSA. Data was entered into and analyzed using SPSS 12.0 for Windows. Odds Ratios (OR) and 9 5\% Confidence Intervals (9 5\% CI) were calculated using Epi Info V3.2.2.

RESULTS: The survey had an overall response rate of $71 \%(7671084) 426(58 \%)$ of 738 respondents indicated that they practiced in institutions with anesthesia training programs (ATP). 29 respondents were excluded due to missing data or report of an alternate practice environment. There were no significant differences between groups with respect to gender or years in practice. ATP respondents had a significantly higher odds of having attended a National Society Meeting (OR 1.9,9 5\% CI 1.4,2.7) and Departmental Rounds (OR 2.8, 9 5\% CI 1.7 , 7) in the past two years, whereas respondents in institutions with no anesthesia training program (NATP) had a higher odds of having attended a Regional Society Meeting (OR 1.4,9 5\% 1.0,1.9) $1 \%$ (3/4 26 bf ATP and 2\% (7/312) of NATP respondents had not attended a CEE in the past two years. There were no significant differences in IR accessed on OSA.

DISCUSSION: This study suggests that there may be significant differences between anesthesiologists in institutions with and without anesthesia training programs in their choice of continued education events but no significant differences in information resources accessed for a specific topic area. This information may assist conference planners in ensuring that their CE content targets the audience most likely to attend their event or alternately suggest the group they may wish to approach to increase their attendance. 